\title{
Numerical Simulation of a Conical Shape Made By Single Point Incremental
}

\author{
J. I.V. de Sena ${ }^{a}$, C. F. Guzman ${ }^{b}$, L. Duchene ${ }^{b}$, A. M. Habraken ${ }^{b}$, R. A. F. Valente ${ }^{a}$, \\ R. J. Alves de Sousa ${ }^{\mathrm{a}}$ \\ ${ }^{a}$ GRIDS Research Group, TEMA Research Unity, Department of Mechanical Engineering, University of Aveiro, \\ Portugal, rsousa@ua.pt,robertt@mec.ua.pt,sena.ilidio@ua.pt \\ ${ }^{b}$ Université de Liège, ArGEnCo Department, MS'Fdivision, Chemin des Chevreuils 1, Liège, 4000, Belgium, \\ anne.habraken@ulg.ac.be, l.duchene@ulg.ac.be,cf.guzman@ulg.ac.be
}

\begin{abstract}
The Single Point Incremental Forming (SPIF) is a manufacturing process in which a sheet is deformed by using a relative small tool without the need of dies.

The current work aims the application of the adaptive remeshing technique developed for shell and extended to 3D "brick" elements in general, and specifically to RESS (Reduced Enhanced Solid-Shell) formulation [1]. The study will be focused on NUMISHEET 2014 benchmark: a cone shape made by SPIF process. The purpose is to use the developed tools to predict the deformed shape and tool-load histories.
\end{abstract}

Keywords: Adaptive Remeshing; Solid Shell finite element; Single Point Incremental Forming.

PACS: PACS numbers: 02.70.DH.

\section{INTRODUCTION}

The research interest in relation to SPIF process has been growing in the last decade, both experimentally and numerically, in the context of sheet metal forming processes. It has been found that the classical theories applied to conventional stamping processes were not suitable for SPIF which has many peculiarities compared to conventional sheet forming processes, such as the apparent increased formability and the deformation mechanisms presented [2]. Due to the vast number of new topics to explore, many works have been carried out, usually covering particular details. From the numerical simulation standpoint, the SPIF process represents a challenge concerning computing time, which tends to be very large. Previous results in the literature $[1,2,3,4,5,6]$ shows that simulated results need a compromise between accuracy and CPU efficiency related with the temporal integration scheme, material law, finite element formulation and mesh size.

\section{ADAPTIVE REMESHING METHOD}

The present section briefly introduces and describes the adaptive remeshing method implemented in the finite element home-made code called LAGAMINE, developed at the University of Liège [3]. This numerical technique leads to the fact that only a portion of the sheet mesh is dynamically refined in the tool vicinity thus following its motion. Doing so, the requirement of initially refined meshes is avoided and consequently, the global CPU time can be reduced. The remeshing criterion adopted is based on the shortest distance between the centre of the spherical tool and the nodes of the finite element. Following this idea, the coarse elements respecting the criterion are deactivated and become a "refined cell" which contains all information about new smaller elements. Each coarse element is dived into a fixed number of new smaller elements defined by the user. The transfer of stress and state variables from the coarse element to the new elements is performed by interpolation method. If the tool is farther from a refined element and the "cell" does not respect the criterion, the new elements are removed and the coarse element is reactivated. However, the shape prediction could be less accurate if the new elements are removed. Consequently, an additional criterion is used to avoid losing accuracy. If the distortion is significant, the refinement remains on the location of the coarse element. As this refinement method does not take into account any transition zone between coarse and fine elements, there are three types 
of nodes: old nodes, free new nodes and constrained new nodes. The constrained nodes are used to allow the structural compatibility of the mesh. The degree of freedom and positions of the constrained nodes on a "cell" edge depend on the two old nodes (masters), which are extremities of this edge. The variable number of degrees of freedom induces modification in the force equilibrium and the tangent stiffness matrix. During the SPIF process simulation many elements are refined and coarsened, so as a result many cells are created and removed. A "linked list" is used as a data structure to insert and remove cells at any point in the list.

\section{REDUCED ENHANCED SOLID-SHELL FINITE ELEMENT (RESS)}

The development of finite element formulations for sheet metal forming has allowed new modelling techniques, such as "Solid-Shell" elements, which combines the main features of shell formulation with a solid topology. The RESS finite element is a hexaedrical element composed by 8 nodes and each node has three degrees of freedom. A combination of Enhanced Assumed Strain method (EAS) and hourglass stabilization in plane, with the use of an arbitrary number of integration points in thickness direction characterize this element. The advantage of RESS integration scheme is the possibility to eliminate the shear locking phenomena, due to the reduced integration in the element plane. The volumetric locking effect is reduced using the EAS method and the reduced integration in the element plane. These choices give more deformation modes to the finite element structure. However, the reduced integration in the element plane provides spontaneous modes of deformation called hourglass phenomena. Consequently, a stabilization scheme is used to eliminate the hourglass effect. This solid-shell element comes from the series of works from Alves de Sousa et al. [1].

The use of a conventional solid element requires several element layers to capture bending effects correctly and multiple layers of finite elements along the thickness increases the computation time. Figure 1 physically presents the advantage of RESS finite element structure compared with different finite elements scheme available in commercial softwares.

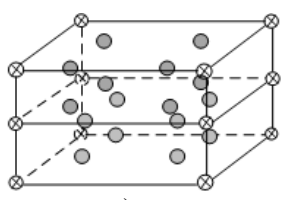

a)

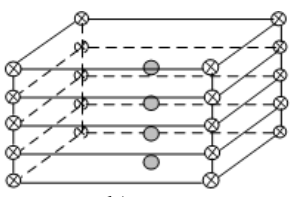

b)

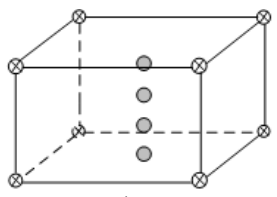

c)

FIGURE 1. Comparison between fully integrated (a), reduced integrated (b) and RESS finite elements (c).

\section{NUMERICAL SIMULATION OF INCREMENTALLY CONICAL SHAPE}

This section presents simulation results of SPIF to produce a conical aluminium part. The sheet material is an AA7075-O aluminium alloy with an initial thickness of $1.6 \mathrm{~mm}$, with the experimental setup and material parameters listed in the documentation available on benchmark proposal.

The isotropic hardening model was described by the Swift Law. To reduce the computation time, a $45^{\circ}$ pie of sheet is modelled and rotational boundary conditions are imposed by displacements on the edges [4]. Two meshes were tested: an initial refined mesh with 5828 elements without the remeshing method and a coarse mesh initially with 410 elements combined with the remeshing technique. The number of integration points through the thickness is 5 and was obtained by preliminary tests using the line-test benchmark [6]. Finally, Coulomb friction coefficient between the tool and sheet is set to 0.01 , value suggested in the benchmark proposal.

The main numerical outputs presented in the following sections are the final shape of the sheet, in a cross-section along the symmetric axis, and the evolution of the tool force in the tool axial direction achieved during the simulation.

Table 1 presents the adaptive remeshing time performance for different level of refinement. It is possible to confirm remeshing advantage even for higher refinement compared with the initially refined mesh proposed, used here as reference. 
TABLE 1. Adaptive remeshing time performance for different level of refinement.

\begin{tabular}{ccccccc}
\hline $\begin{array}{c}\text { № of nodes } \\
\text { per edge (n) }\end{array}$ & CPU time & $\begin{array}{c}\text { CPU } \\
\text { time } \\
\text { Ratio }\end{array}$ & № of Steps & $\begin{array}{c}\text { № of } \\
\text { iterations }\end{array}$ & $\begin{array}{c}\text { Initial no of } \\
\text { elements }\end{array}$ & $\begin{array}{c}\text { Final no of } \\
\text { elements }\end{array}$ \\
\hline 1 & $59 \mathrm{~m}: 46 \mathrm{~s}$ & 0.06 & 2072 & 9509 & 410 & 1106 \\
2 & $2 \mathrm{~h}: 32 \mathrm{~m}: 25 \mathrm{~s}$ & 0.16 & 2290 & 9689 & 410 & 2228 \\
3 & $7 \mathrm{~h}: 4 \mathrm{~m}: 43 \mathrm{~s}$ & 0.45 & 2296 & 9606 & 410 & 3610 \\
4 & $9 \mathrm{~h}: 35 \mathrm{~m}: 55 \mathrm{~s}$ & 0.61 & 2297 & 9642 & 410 & 5860 \\
Reference & $15 \mathrm{~h}: 39 \mathrm{~m}: 1 \mathrm{~s}$ & 1 & 5263 & 22443 & 5828 & 5828 \\
\hline
\end{tabular}

\section{Force Prediction}

The force prediction presented in Figure 2 is the axial tool force component (Fz). To simplify the comparison, the numerical force evolution is replaced by the average calculation when the tool is in the central positions of each contour, to avoid inaccuracy from boundary conditions. The axial force can be analytically predicted by a generalized formula described by Aerens et al. [5]. The analytical force calculation was used to anticipate and verify if the achieved forces during the numerical simulations are acceptable.

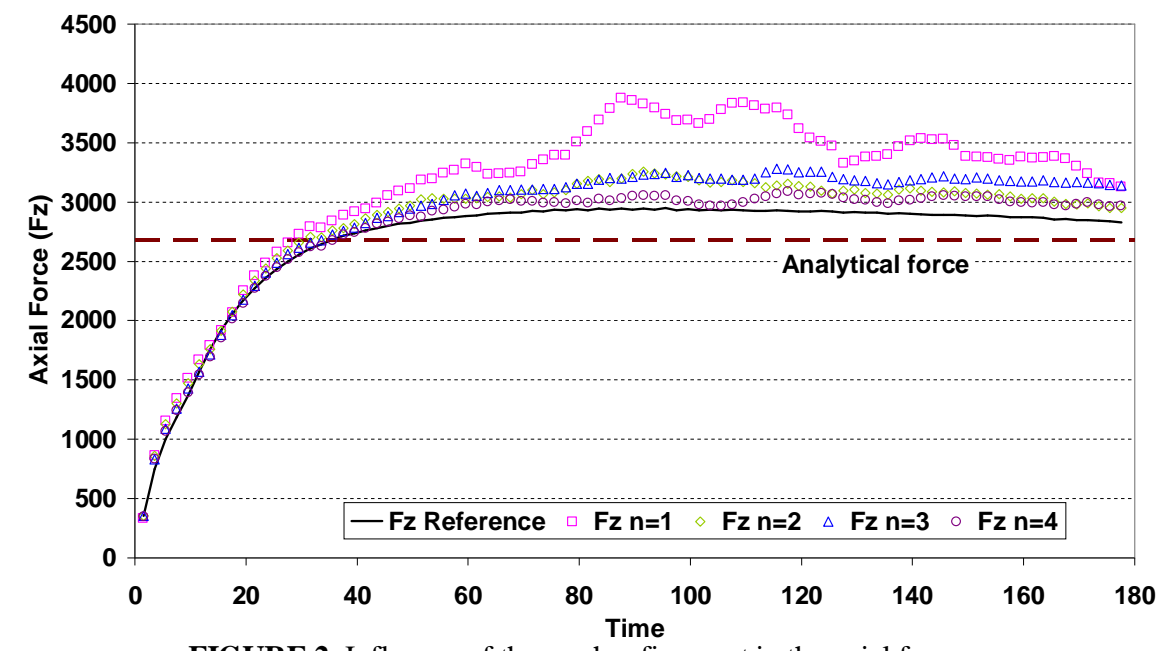

FIGURE 2. Influence of the mesh refinement in the axial force.

The results are quite similar between the reference case and the remeshing process using high number nodes per edge. The oscillations of the force are due to the penalty method and the contact. They are higher with remeshing because the mesh used is coarser than the reference one. It is notable that the results are sensitive to the variation of the number of new smaller elements per coarse element.

\section{Shape Profile and Thickness}

The numerical shape is extracted from the middle section of the pie mesh used within the FE model to avoid inaccuracy due to boundary conditions. Figure 3 indicates a good prediction of the shape for the different levels of remeshing when compared with the reference mesh. The thickness prediction has analogous results compared to the reference thickness. 


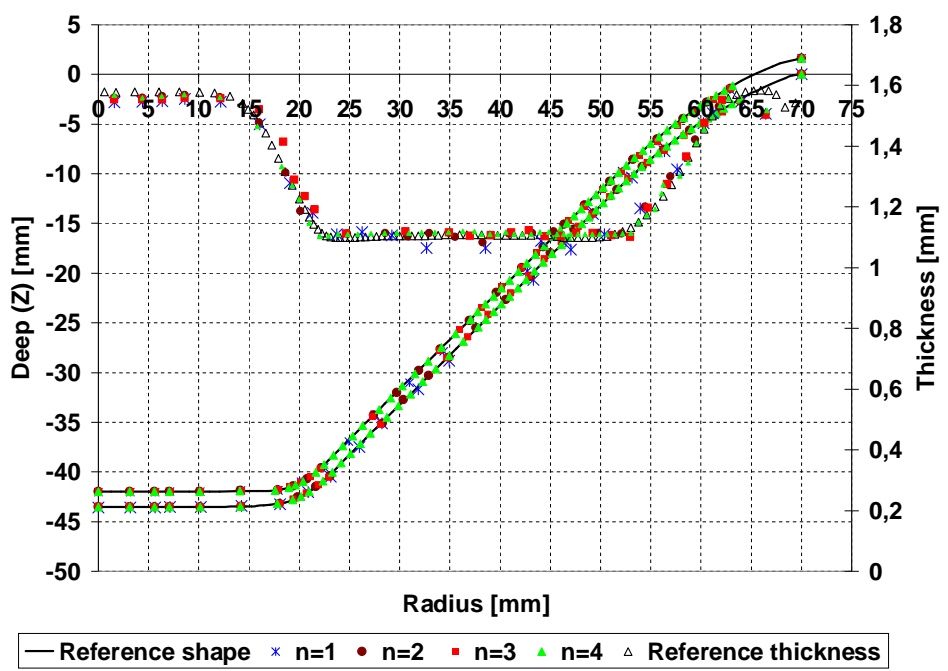

FIGURE 3. Final shape and thickness prediction.

\section{FINAL CONSIDERATIONS}

The current article studies the influence of the refinement parameters used in an adaptive remeshing procedure in the prediction of the tool force and the shape for a conical component. This remeshing method has the advantage to strongly decrease the number of nodes and inducing CPU time reduction while giving quite similar results as using an initially refined mesh.

The use of 3D finite elements, when compared to shell elements, allows for a direct consideration of thickness variations, full 3D constitutive laws and evaluation of all components of the stress field.

\section{ACKNOWLEDGMENTS}

The authors would like to gratefully acknowledge the support given by Portuguese Science Foundation (FCT) under the grant SFRH/BD/71269/2010 (J. I. V. Sena).

As Research director, A.M. Habraken would like to thank the Fund for Scientific Research (F.R.S FNRS, Belgium) for its support.

\section{REFERENCES}

1. J.I.V. Sena, R.J. Alves de Sousa, R.A.F. Valente, "On the use of an EAS solid-shell element to simulate incremental formally processes", Engineering Computations, Vol. 28 No. 3, 2011, pp. 287-313.

2. P. Eyckens, J. Del-lero Moreau, J. Duflou, A. Van Bael, P. Van Houtte, "MK Modelling of sheet formability in the incremental sheet forming process, taking into account through-thickness shear", International Journal of Material Forming, Vol.2, 2009, pp.379-382.

3. C. Lequesne., C. Henrard, C. Bouffioux, J. Duflou, and A.M. Habraken, "Adaptive Remeshing for Incremental Forming Simulation", Proceedings of the NUMISHEET $20087^{\text {th }}$ International Conference and workshop on numerical simulation of 3D sheet metal forming processes, Institute of Virtual Manufacturing ETH Zurich, September 1-5, Interlaken, Switzerland.

4. C. Bouffioux, P. Eyckens, C. Henrard, R. Aerens, A. Van Bael, H. Sol, J. R. Duflou and A. M. Habraken, "Identification of material parameters to predict single point incremental forming forces", International Journal of Material Forming, Vol. 1, 2008, pp.1147-1150.

5. R. Aerens, P. Eyckens, A. Van Bael, J. Duflou, "Force Prediction for Single Point Incremental Forming Deduced from Experimental and FEM Observations", International Journal of Advanced Manufacturing Technology, Vol.46, 2010, pp.969-982.

6. C. Henrard, "Numerical Simulations of the Single Point Incremental Forming Process", Ph.D. Thesis, Université de Liège, Belgium, 2008. 